Revue d'histoire de l'Amérique française

सES REVUE D.HISTOIRE DE L'AMÉRIQUE FRANÇAISE

\title{
Aperçu sur le commerce et le crédit à Québec 1820-1830
}

\section{George Bervin}

Volume 36, numéro 4, mars 1983

URI : https://id.erudit.org/iderudit/304094ar

DOI : https://doi.org/10.7202/304094ar

Aller au sommaire du numéro

Éditeur(s)

Institut d'histoire de l'Amérique française

ISSN

0035-2357 (imprimé)

1492-1383 (numérique)

Découvrir la revue

Citer cet article

Bervin, G. (1983). Aperçu sur le commerce et le crédit à Québec 1820-1830.

Revue d'histoire de l'Amérique française, 36(4), 527-551.

https://doi.org/10.7202/304094ar d'utilisation que vous pouvez consulter en ligne.

https://apropos.erudit.org/fr/usagers/politique-dutilisation/ 


\section{APERÇU SUR LE COMMERCE ET LE CRÉDIT À QUÉBEC 1820-1830*}

GEORGE BERVIN

Département d'histoire

Université de Montréal

\section{INTRODUCTION}

Au cours de la période 1820-1830, le Bas-Canada se situe dans une ère de capitalisme commercial qui a déjà atteint son rythme de croisière. À cette époque, l'économie coloniale est «bien accrochée au marché atlantique et aboutée au continent nord-américain par les flux de biens, de capitaux, de personnes et d'informations» ${ }^{1}$. Et les indices du passage d'une économie de capitalisme commercial à un capitalisme industriel commencent à se manifester timidement.

À l'intérieur de cette économie, une nouvelle génération de marchands a fait son entrée: les marchands-négociants ${ }^{2}$. Ceux-ci ne se limitent plus à une activité commerciale spécifique, car leur caractéristique première consiste en la diversification et au volume de leurs activités. Ces marchands-négociants font preuve d'une certaine agressivité au niveau économique, dans la mesure où, durant la période $1820-1830$, ils se révèlent massivement présents dans les secteurs les plus rentables. Ces secteurs, pour ne nommer que les plus importants, sont le commerce du bois, l'importexport, la construction navale et domiciliaire, le commerce des biens de consommation et le système de crédit tel que défini dans la partie II du présent article. C'est grâce au «boom» survenu dans

* Nous tenons à remercier tout particulièrement monsieur Jean-Pierre Wallot pour ses commentaires et suggestions, ainsi que France Galarneau, du Dictionnaire biographique $d u$ Canada, pour son aide tout au long de la rédaction de cet article. Cette recherche se situe dans le cadre d'un projet dirigé par Jean-Pierre Wallot, Gilles Paquet, Jean-Pierre Hardy et Thierry Ruddell, portant sur l'histoire économique et la culture matérielle du Bas-Canada dans la première moitié du XIXe siècle. Ce projet a été rendu possible grâce à des subventions du fonds FCAC, du CRSHC et du Musée National de l'Homme.

1 Gilles Paquet et Jean-Pierre Wallot, «Sur quelques discontinuités dans l'expérience socio-économique du Québec: une hypothèse», Revue d'histoire de l'Amérique française, 35, 4 (mars 1982): 483-521.

${ }_{2}$ Pour une définition plus détaillée du marchand-négociant et de son mode de vie, consulter: George Bervin, «Espace physique et culture matérielle du marchand-négociant à Québec (1820-1830)», Bulletin d'histoire de la culture matérielle, 14 (Musée National de l'Homme, Ottawa): printemps 1982. 
ces différents secteurs que peut s'expliquer la consolidation de cette bourgeoisie commerçante.

Notre étude, qui se situe à l'intérieur de cette phase de capitalisme commercial, se compose de deux volets. Le premier traite du commerce de gros et de détail, et le deuxième aborde le système de crédit. La description du commerce et du crédit fait apparaître en filigrane une société de classes. On la voit poindre avec plus d'insistance à cause d'un commerce d'objets de luxe qui n'est pas à la portée de tous, mais aussi à cause de la monopolisation du crédit par une catégorie sociale bien identifiée à l'intérieur de la ville de Québec. Cette catégorie est constituée principalement de marchands-négociants.

En fait, les marchands-négociants sont des personnages qu'on ne peut pas confondre avec les petits commerçants de quartier ou de village, tant sur le plan des affaires que sur le plan social. La diversification de leurs capitaux se manifeste, entre autres, par la propriété de magasins de gros et de détail, et de manufactures ${ }^{3}$. Au niveau de la finance, ils se retrouvent presque les seuls à prêter avec intérêt à des particulliers des montants supérieurs à $£ 1000$ courant. Un autre caractère leur est spécifique, soit la variété de leurs relations d'affaires. En plus d'avoir de tels rapports tant dans le Bas que dans le Haut-Canada, ils entretiennent également des liens privilégiés avec des marchands des États-Unis et d'Europe, et profitent d'un réseau d'informations ${ }^{4}$ auquel les petits commerçants n'ont pas accès.

Les marchands-négociants sont aussi de gros propriétaires fonciers. À la campagne, leur présence se traduit par la possession de vastes étendues de terre qui leur permet d'exercer une intense activité spéculative. À l'intérieur de la ville, ils sont propriétaires

3 La manufacture dont il est question ici se résume à un vaste entrepôt où se trouvent réunis plusieurs travailleurs qui, sous la direction d'un contremaître, produisent un bien donné dans le but de répondre rapidement à la demande du marché. Dans ce type de manufacture, il n'y a pas, à proprement parler, de division du travail ni de mécanisation poussée des appareils de production. La manufacture de tabac du marchand-négociant John Reinhart, dont nous reproduisons le stock de production au tableau III, en est un exemple. À l'intérieur de cette manufacture, on peut quand même noter une certaine présence de la mécanique et cela constitue, selon nous, le prélude à une mécanisation plus avancée. Ainsi, on retrouve chez Reinhart sept moulins à moudre le tabac, une meule montée, quatre grandes roues de fer pour servir à un moulin, deux presses pour presser le tabac, une grande boîte en bois pour «manufacturer» le tabac, un réservoir contenant 12000 livres de fleur de tabac, environ 56 barriques dont chacune contient 400 livres de tabac, et un tas d'autres outils. Tout le travail se fait sous la direction d'un contremaître, et le notaire mentionne dans l'inventaire après décès, qu'il s'agit d'une «manufacture», ANQ-Q, not. Louis Panet, no 263: 11-6-1821.

Pour comprendre l'importance de ces réseaux d'informations qui favorisent les gros marchands, voir en particulier le chapitre V de l'ouvrage de Gilles Paquet et JeanPierre Wallot, Patronage et pouvoir dans le Bas-Canada (1794-1812). Un essai d'économie historique, (Montréal, Les Presses de l'Université du Québec, 1973). 
de multiples bâtisses et emplacements commerciaux et résidentiels, qu'ils mettent en location ou en vente. On peut dire que les marchands-négociants forment l'aristocratie du milieu des affaires dans la ville de Québec entre 1820 et 1830, et qu'ils représentent la branche dominante de cette bourgeoisie commerçante.

Les principales recherches portant sur les différents secteurs d'investissement de ces marchands-négociants ont revêtu jusqu'à présent un caractère plus général que particulier, c'est-à-dire se situant dans un cadre englobant l'ensemble de l'histoire économique $^{5}$. À ce stade-ci, nous désirons tenter d'élargir et d'approfondir la problématique sur deux champs d'activité économique (commerce et crédit) des marchands-négociants de Québec. Évidemment, rien n'est définitif dans cette esquisse qui vise à ouvrir de nouvelles pistes de recherche qu'il conviendra d'explorer de façon plus systématique.

\section{SOURCES ET MÉTHODES}

Nos sources d'informations reposent principalement sur les archives notariales, la Gazette de Québec (des années 1820 à 1830), les «Ledgers» de la Banque de Québec et les Quebec Almanachs. Parmi les archives notariales, nous avons surtout utilisé les inventaires après décès, plus spécialement ceux des marchandsnégociants pour qui un tel inventaire a été fait. On retrouve dans les stocks de marchandises énumérés dans ces inventaires, ainsi que dans la Gazette de Québec, presque toutes les gammes de produits destinés aux marchés de gros et de détail. Dans la partie des inventaires portant sur les créances (dettes actives et passives), toutes les couches socio-professionnelles de la ville de Québec sont représentées, des petits commerçants de quartier aux gros marchands les plus actifs (comme par exemple George Pozer, John Goudie,

5 Cette affirmation concerne en particulier la ville de Québec. Pour Montréal, Gérald J. J. Tulchinsky a démontré comment la bourgeoisie d'affaires montréalaise entre 1837 et 1853 s'est développée et a entrepris la diversification de ses activités économiques. L'auteur a reconstitué le caractère, les motivations et les intérêts de cette bourgeoisie d'affaires (The River Barons. Montreal Businessmen and the Industry and Transportation, Toronto, University of Toronto Press, 1977, 307 p.). De même, il faut retenir l'étude de José Igartua sur les commerçants et négociants de Montréal dans la seconde moitié du XVIIIe siècle ("The Merchants of Montreal at the Conquest: Socio-Economic profile», Histoire sociale, 8, 16 (nov.-déc. 1975): 275-293). Pour sa part, Fernand Ouellet ne fait pas tellement de distinctions entre les différents membres du groupe des marchands dans l'ensemble du Bas-Canada, et n'identifie pas systématiquement les activités de chacune des composantes du groupe (Éléments d'histoire sociale du Bas-Canada, Montréal, HMH Ltée, 1972, p. 189; Histoire économique et sociale du Québec, 1760-1850, Montréal, Fides, 1971). Les professeurs Paquet et Wallot abordent eux aussi le milieu des affaires et en qualifient les membres les plus importants de «grande bourgeoisie d'affaires», mais eux non plus n'en décrivent pas les différentes strates d'activité («Groupes sociaux et pouvoir: le cas canadien au tournant du XIXe siècle», $R H A F, 27,4$ (mars 1974): 539). 
James McCallum, William Price), en passant par les hauts fonctionnaires de l'État.

Pour la partie de notre étude touchant le crédit, en plus de la section des dettes actives et passives des inventaires après décès, nous nous sommes servi d'environ une centaine d'obligations et de quittances passées entre les marchands, entre ces derniers et les banques, et entre les marchands et le reste de la population, ainsi que des «Ledgers» de la Banque de Québec. Ces différents actes notariés, ainsi que le livre comptable de cette banque, permettent de brosser un tableau du système de crédit dans la ville de Québec.

Au niveau méthodologique, après avoir constitué un échantillon de 20 inventaires après décès de marchands-négociants ${ }^{6}$, nous avons analysé les stocks de marchandises inventoriées, ainsi que la section des dettes actives et passives. Dans un second temps, à chaque fois qu'il nous fallait présenter un fait ou développer une analyse, nous avons retenu l'exemple de trois ou quatre marchands-négociants typiques de l'ensemble de notre échantillon, de manière à illustrer la tendance générale ${ }^{7}$.

\section{1 - Le commerce de gros et de détail}

Lorsqu'on se penche sur le commerce à Québec au cours de la décennie 1820 , on ne peut qu'être frappé par son aspect international et par l'abondance des produits disponibles sur le marché. Si on retient pour indice la provenance diversifiée des produits importés, il semble clair que la ville fait partie du circuit commercial international ${ }^{8}$. Le port ne sert pas alors uniquement à exporter les ressources naturelles, mais constitue un maillon du commerce international et du commerce interne.

Avant de donner quelques chiffres qui permettront de saisir l'ampleur des stocks, nous allons d'abord faire un survol de ce qu'on trouve sur le marché et voir quels sont les secteurs dominants.

Les données du tableau I illustrent clairement que le secteur alimentaire prévaut sur la plupart des autres secteurs. Les aliments constituent respectivement $36 \%, 44 \%, 17 \%$ (dans le cas de $\mathrm{C}$, le

\footnotetext{
6 Ces inventaires se trouvent aux Archives nationales du Québec à Québec
(ANQ-O), plus précisément dans les minutiers des notaires suivants: Jean Bélanger (1823); L. T. Besserer (1822); A. Campbell (1822); C. M. De Foy $(1825,1830)$; E. Glackmeyer (1827); E.B. Lindsay (1825, 1829); Thomas Lee $(1822,1825)$; L.T. MacPherson (1830); Louis Panet $(1820,1821)$ et Joseph Planté $(1824,1825)$.

7 Mais nous avons toujours tenu compte dans notre analyse de l'ensemble de notre échantillon, soit des 20 inventaires et des données recueillies dans la Gazette de Québec.

8 Gilles Paquet et Jean-Pierre Wallot, "Aperçu sur le commerce international et les prix domestiques dans le Bas-Canada (1793-1812)», RHAF, 21, 3 (déc. 1967): 447-473.
} 
pourcentage peu élevé peut s'expliquer par la place prépondérante que tient la quincaillerie dans le stock de ce marchand) et $41 \%$ de la valeur des marchandises figurant au tableau I. Si l'on se permet d'inclure les spiritueux, cette domination s'accentue encore et s'élève à environ $50 \%$ de la valeur totale des marchandises en vente.

\section{TABLEAU I}

\section{Répartition des stocks de marchandises en magasin}

En $\%$ de la valeur totale

Principales composantes (en chiffres absolus)

\begin{tabular}{lrrrr}
\hline & A & B & C & D \\
\hline Aliments & 36 & 44 & 17 & 41 \\
Spiritueux & 12 & 14 & 2 & 21 \\
Produits domestiques & 0 & 7 & 1 & 1 \\
Chasse et pêche & 9 & 4 & 1 & 0 \\
Ustensiles-vaisselle & 0 & 15 & 5 & 5 \\
Quincaillerie & 14 & 5 & 71 & 3 \\
Habillement-tissus-lingerie & 29 & 4 & 1 & 26 \\
Divers & 0 & 7 & 2 & 3 \\
Valeur totale des marchandises & & & & \\
(en £ courant) & 1444 & 1710 & 1857 & 366 \\
(en \#) & 34656 & 41040 & 44568 & 8784 \\
\hline
\end{tabular}

Légende: A- Marchandises dans les magasins de Michel Borne à Québec (Source: ANQ-Q, not. Charles Maxime De Foy, no 1201, 25-1-1830)

B- Marchandises dans le magasin de Frederick Limpp à Québec (Source: ANQ-Q, not. Louis Panet, no 45, 29-1-1820)

C- Marchandises dans le magasin de John McCord à Québec (Source: ANQ-Q, not. Archibald Campbell, no 661, 29-11822)

D- Marchandises dans le magasin de Louis Noël à Québec (Source: ANQ-Q, not. Louis Thomas Besserer, 24-4-1822).

La plupart des grands marchands ont des intérêts dans le secteur alimentaire. Ne voit-on pas un entrepreneur comme William Price, identifié au commerce du bois, vendre des produits comestibles $^{9}$, un John Goudie, identifié au secteur de la construction navale, tenir une boulangerie et une boucherie, en plus d'être propriétaire d'un magasin général ${ }^{10}$. Le tableau I reflète donc une

${ }^{9}$ La Gazette de Québec, 22 juin 1826.

10 ANQ-Q, not. Thomas Lee, no 1825, 10-1-1825. 
situation presque généralisée, ou si l'on veut, la conjoncture économique de l'époque. Il s'agit là d'un type de commerce générateur de profits substantiels. Ceci vaut particulièrement dans la ville où la population est obligée de tout se procurer pour se nourrir, contrairement à la campagne environnante où les habitants peuvent s'autosuffire davantage.

Ces sources de profits dans le secteur alimentaire sont liées dans une large mesure à deux paramètres. Le premier, que nous qualifions de «croissance progressive normale» de la population de la ville, consiste en l'augmentation du nombre de travailleurs (en partie liée au développement de certains corps de métiers nettement spécialisés, en particulier dans la construction navale et domiciliaire) ${ }^{11}$, puis du nombre de journaliers formés en grande partie de gens des campagnes environnantes, auxquels s'ajoutent les nouveaux artisans de toutes sortes et les immigrants. Le deuxième paramètre, ou «l'augmentation provisoire», c'est tout simplement la partie «mobile» de la population de la ville, celle qui vient se greffer provisoirement à la population sédentaire. Cette situation vient du fait que Québec est une ville de transit pour les immigrants qui iront s'installer ailleurs, soit à Montréal, soit dans le Haut-Canada et éventuellement aux États-Unis. Par ailleurs, la ville abrite une forte garnison militaire, ainsi que des draveurs, bûcherons et marins de passage. C'est donc dire qu'il y a un grand nombre d'individus qui à une certaine période font grossir artificiellement l'effectif de la population de la ville ${ }^{12}$. Toute cette population normale et provisoire possède un point en commun: elle doit se nourrir, se vêtir, se loger. C'est à ce moment qu'interviennent les marchands qui voient là une source de profits intéressants et une façon de diversifier leurs investissements.

Le tableau I renferme aussi des secteurs différents de l'alimentation qui tiennent une place non négligeable dans les stocks. Ainsi, celui de la quincaillerie compte pour $14 \%, 5 \%, 71 \%$ et $3 \%$ des marchandises totales; puis, suit celui de l'habillement-tissuslingerie, avec $29 \%, 4 \%, 1 \%$ et $26 \%$ des marchandises totales. Dans une certaine mesure, l'intérêt des commerçants pour ces secteurs repose sur les mêmes considérations que celles dont nous avons précédemment fait mention.

11 En ce qui a trait à la question domiciliaire, voir Jacques Bernier, «La construction domiciliaire à Québec, 1810-1820», RHAF, 31, 4 (mars 1978): 547-561, et Geneviève G. Bastien et al., Inventaire des marchés de construction des archives civiles de Québec, 1800-1870 (3 vol., Ottawa, 1975). Dans ce dernier cas, les auteurs ont répertorié la plupart des marchés de construction (contrats) concernant Québec et sa région, disponibles aux ANQ-Q.

12 Fernand Ouellet, «Structures des occupations et ethnicité dans les villes de Québec et de Montréal (1819-1844)", in Éléments d'histoire sociale du Bas-Canada (Montréal, Éditions Hurtubise HMH, 1972). 
Si les secteurs de la quincaillerie et de l'habillement constituent un pourcentage raisonnable des stocks de marchandises et dénotent une tendance à la hausse au cours de la période 18201830 , tel n'est pas le cas pour le secteur des produits domestiques, qui demeurera presque stationnaire durant cette période. Le tableau I montre que ce secteur, avec respectivement $0 \%, 7 \%, 1 \%$ et $1 \%$ du total des marchandises, détient une place assez mince sur le marché de gros et de détail, et que les articles les plus vendus sont le tabac, l'huile à brûler et les chandelles.

Le tableau II permet d'identifier sept catégories de produits et contient une description de chacune d'elles. Mais, d'où viennent ces produits? Selon leur nature, ils viennent directement et en grande quantité d'Angleterre d'abord (sauf dans l'alimentation où les approvisionnements sont diversifiés: Bas-Canada, HautCanada, États-Unis et parfois Grande-Bretagne), souvent en ligne droite des possessions anglaises aux Antilles (Jamaïque, SainteLucie, Trinidad, etc.), de Cuba et du Haut-Canada. Nous n'avons pas retracé de produits en provenance des possessions françaises aux Antilles (Martinique, Guadeloupe), mais plusieurs de France. Ce sont dans ce cas des produits qui ne sont pas de première nécessité: du champagne, du vin, des olives, des prunes, du vinaigre, des livres, des estampes, des tableaux. On retrouve aussi dans le commerce des produits qui viennent des États-Unis, d'Espagne (produits destinés à une clientèle spécifique: vins, noix, cigares, pièces de mobilier). D'autres sont importés de Hollande, du Brésil (entre autres du bois), de Russie (des tissus), de Chine (du thé), du Portugal et de la Confédération germanique.

La variété et l'abondance des produits vendus nous amènent à constater qu'en temps normal, la population de la ville se trouve en mesure de se procurer (en autant qu'elle en ait les moyens financiers) une grande variété de denrées en quantité suffisante. Mis à part les magasins qui regorgent de marchandises, la Gazette de Québec signale à presque chacune de ses parutions l'arrivée dans le port d'au moins un navire ayant à son bord des stocks de marchandises sèches et comestibles destinées à la vente et cela, évidemment, quand la saison le permet. Une partie de ces marchandises se retrouvent plus tard aux marchés de la haute ville et à ceux de la basse ville. Le reste est envoyé dans d'autres régions, comme Montréal et même dans le Haut-Canada.

$\mathrm{Au}$ tableau I, on remarquera la très grande place que tiennent les produits manufacturés dans les stocks de marchandises; la Gazette de Québec de la période allant de 1820 à 1830 confirme cette observation. En tête de liste se trouvent les ustensiles et la vaisselle, la quincaillerie, suivis des textiles, comprenant les tissus 
TABLEAU II

Identification de sept catégories de «produits-denrées» dans le commerce à Québec (1820-1830)

\begin{tabular}{|c|c|c|c|c|c|c|}
\hline Aliments & Spiritueux & $\begin{array}{l}\text { Produits } \\
\text { domestiques }\end{array}$ & $\begin{array}{l}\text { Chasse et } \\
\text { pêche }\end{array}$ & $\begin{array}{l}\text { Ustensiles- } \\
\text { vaisselle }\end{array}$ & $\begin{array}{l}\text { Quincail- } \\
\text { lerie }\end{array}$ & $\begin{array}{l}\text { Habillement- } \\
\text { tissus-lingerie }\end{array}$ \\
\hline $\begin{array}{l}\text { amandes } \\
\text { beurre } \\
\text { biscuits } \\
\text { café } \\
\text { cassonade } \\
\text { céréales } \\
\text { chocolat } \\
\text { cornichons } \\
\text { épices } \\
\text { farine } \\
\text { fromage } \\
\text { huile } \\
\text { lard } \\
\text { mélasse } \\
\text { menthe (essence) } \\
\text { miel } \\
\text { moutarde } \\
\text { navets } \\
\text { noix } \\
\text { oignons } \\
\text { patates } \\
\text { piments } \\
\text { pois } \\
\text { poissons } \\
\text { sel } \\
\text { sucre } \\
\text { thé } \\
\text { viandes } \\
\text { vinaigre }\end{array}$ & $\begin{array}{l}\text { bière } \\
\text { brandy } \\
\text { champagne } \\
\text { cognac } \\
\text { eau-de-vie } \\
\text { gin } \\
\text { grog } \\
\text { rhum } \\
\text { vin } \\
\text { whisky }\end{array}$ & $\begin{array}{l}\text { chandelles } \\
\text { cirage } \\
\text { huile à brûler } \\
\text { indigo } \\
\text { papier } \\
\text { savon } \\
\text { tabac }\end{array}$ & $\begin{array}{l}\text { ancres } \\
\text { cordages } \\
\text { corde à voile } \\
\text { crochets } \\
\text { fusils } \\
\text { hameçons } \\
\text { lignes } \\
\text { pièges } \\
\text { pierres à fusil } \\
\text { plombs à tirer } \\
\text { poudre à tirer } \\
\text { voile }\end{array}$ & $\begin{array}{l}\text { assiettes } \\
\text { barriques } \\
\text { bols } \\
\text { bouilloires } \\
\text { bouteilles } \\
\text { cafetières } \\
\text { carafes } \\
\text { chaudières } \\
\text { chaudrons } \\
\text { cruches } \\
\text { cuillers } \\
\text { cuves } \\
\text { fourchettes } \\
\text { gobelets } \\
\text { jarres } \\
\text { moules } \\
\text { moulins } \\
\text { plats } \\
\text { plateaux } \\
\text { pots } \\
\text { quart } \\
\text { salières } \\
\text { sauciers } \\
\text { service à dîner } \\
\text { soucoupes } \\
\text { sucrier } \\
\text { tasses } \\
\text { terrines } \\
\text { théières } \\
\text { tonneaux } \\
\text { verres }\end{array}$ & $\begin{array}{l}\text { acier } \\
\text { balances } \\
\text { bêches } \\
\text { bouvets } \\
\text { cadenas } \\
\text { ciseaux } \\
\text { clous } \\
\text { colle } \\
\text { corde } \\
\text { égoïnes } \\
\text { fil de fer } \\
\text { galères } \\
\text { goudron } \\
\text { haches } \\
\text { marteaux } \\
\text { mastic } \\
\text { peinture } \\
\text { pelles } \\
\text { pentures } \\
\text { pinceaux } \\
\text { pioches } \\
\text { planches } \\
\text { poêles } \\
\text { poids } \\
\text { rabots } \\
\text { râteaux } \\
\text { scies } \\
\text { serrures } \\
\text { térébentine } \\
\text { truelles } \\
\text { varlopes } \\
\text { vis } \\
\text { vitres } \\
\text { vrilles }\end{array}$ & $\begin{array}{l}\text { bas } \\
\text { bonnets } \\
\text { bottes } \\
\text { boutons } \\
\text { caleçons } \\
\text { châles } \\
\text { chapeaux } \\
\text { chaussures } \\
\text { corsets } \\
\text { couvertures } \\
\text { dentelles } \\
\text { draps } \\
\text { fil } \\
\text { gants } \\
\text { habits } \\
\text { laine } \\
\text { manteaux } \\
\text { mouchoirs } \\
\text { rubans } \\
\text { tissus }\end{array}$ \\
\hline
\end{tabular}


de toutes sortes et l'habillement. Il ne faut pas non plus oublier le secteur de la métallurgie, avec les poêles, les clous, les outils de forgeron, de construction, d'agriculture et de menuiserie (secteur intégré à la quincaillerie). Autres produits manufacturés, le goudron, la peinture, les plombs et la poudre à tirer, ainsi que les fusils. On peut donc constater que la plupart des marchandises en magasin sont constituées de produits finis ou transformés et que ce sont dans l'ensemble des stocks d'importation.

Le commerce de gros est étroitement lié à celui de détail, particulièrement dans le cas des gros marchands. Dans leur magasin ou leur entrepôt, il n'est pas facile de faire une nette distinction entre les produits réservés à la vente à de petits commerçants, et ceux prévus pour être détaillés en magasin. Cela est, en effet, rendu difficile par la très grande quantité de marchandises en stock. Cette situation se présente chez plusieurs marchandsnégociants qui possèdent un magasin de gros et/ou de détail.

Ainsi, dans le magasin de Frederick Limpp ${ }^{13}$, la présence de plus de 500 gallons de spiritueux, de 11 barils de sucre et de près de 47000 clous de diverses dimensions, pour ne citer que ces quelques marchandises, donne à penser que ces articles ne sont pas seulement destinés à être écoulés dans son magasin, mais aussi à être vendus en gros à d'autres marchands de moindre envergure. Par contre, il ne s'avère pas aisé de mesurer à l'intérieur d'un même produit la quantité qui doit être vendue en gros et celle qui le sera au détail, car l'inventaire ne fournit que très rarement cette précision. Donc, à en juger par l'imposant stock de marchandises détenu par certains marchands-négociants, il n'est pas difficile de croire qu'une partie au moins sera orientée vers la vente de gros ${ }^{14}$. Ainsi, le tableau III, qui concerne la manufacture de tabac de John Reinhart, met en évidence ce fait, car on imagine mal qu'une

\footnotetext{
13 ANQ-Q, not. Louis Panet, no 45, 29-1-1820, Inventaire après décès de Frederick Limpp.

14 Quelques exemples tirés des stocks de marchandises de certains commerçants nous renseignent sur l'abondance des produits disponibles sur le marché. Dans le magasin de Michel Borne: 3986 verges de tissus et 270 paires de bottes de toutes sortes, 561 hameçons, 600 plumes à écrire, 500 pierres à fusil et 9 tonnes de rhum. Dans le magasin de Frederick Limpp: 4874 livres de gruau, 4300 livres de beurre, 267 gallons de mélasse, 7300 livres de sucre, 4675 livres de tabac, 140 boîtes plus 4 barils de cirage à chaussures, 600 douzaines de pièces de vaisselle de toutes sortes. Dans le magasin de John McCord: 172 douzaines de cadenas, 106 boîtes de vitre plus 828 morceaux de vitre, près d'un million de clous de toutes sortes, 60 douzaines de pinceaux, 1376 paires de pentures, 93 haches, 269 vrilles. Dans le magasin de Louis Noël: 29 douzaines de ciseaux, 576 gallons de spiritueux, 3200 livres de chocolat, 2000 livres de sucre. On comprendra que l'énumération de ces différents articles ne reflète qu'une faible partie de la composition des stocks de marchandises de ces quatre marchands. Elle permet toutefois de saisir l'ampleur du commerce dans la ville, à cause justement de cette accumulation extraordinaire de produits en vente qui témoigne de la forte demande pour de tels produits.
} 


\section{TABLEAU III}

Composition du stock de production: manufacture de tabac de John Reinhart

\begin{tabular}{lll} 
Composantes & Quantité/état & $\begin{array}{l}\text { Valeur en } \\
£ \text { courant }\end{array}$ \\
\hline Tabac & 28 barils, $400 \mathrm{lbs} / \mathrm{ba}$. & 420 \\
Tabac (en fermentation) & 28 barils, 300 lbs/ba. & 315 \\
Fleur de tabac & 1 baril de $550 \mathrm{lbs}$ & $10 / 6 / 3$ \\
Fleur de tabac & $12000 \mathrm{lbs}$ dans réservoir & 450 \\
Fleur de tabac & 15 barils & $77 / 6 / 10$ \\
Fleur de tabac & 2 boîtes (1 375 lbs) & $25 / 15 / 7$ \\
\hline Total & & $£ 1298 / 8 / 8$ \\
& & $(31162 \# 8$ sols) \\
\hline
\end{tabular}

Source: ANQ-Q, not. Louis Panet, no 263, 11-6-1821.

quantité aussi considérable de tabac ne soit destinée qu'à sa tabagie ${ }^{15}$.

D'autre part, une certaine quantité de produits manufacturés sont vendus à des commerçants à l'extérieur de la ville de Québec. Ceci est confirmé par des annonces parues dans la Gazette de Qué$b e c$, dans lesquelles certains manufacturiers de tabac prennent la peine de mentionner qu'ils répondront avec diligence à toute commande provenant des commerçants de la campagne.

Les ventes à l'encan, pour leur part, sont généralement destinées aux marchands détenant un commerce, à cause des quantités énormes de marchandises mises aux enchères. De plus, l'annonce fait quelquefois mention de l'obligation de payer en argent comptant. Dans une telle situation, on peut présumer que seuls les marchands (autrement dit les revendeurs des cargaisons qui sont débarquées dans le port) disposent du numéraire pour l'achat de lots considérables. D'ailleurs, avant l'arrivée de la cargaison, les encanteurs font leur publicité dans les journaux pour prévenir les commerçants de la venue imminente de telle ou telle sorte de marchandises. Ces indices laissent supposer que ces produits sont écoulés en gros, donc vendus à certains marchands qui, à leur tour, revendent ces marchandises à plusieurs petits commerçants, ou encore au détail. Parfois encore, ils les achètent dès leur arrivée et

${ }^{15}$ Le stock de production qui apparaît dans le tableau III ne représente peut-être pas tout le volume véritable dont dispose John Reinhart, car il possède, en association, une autre manufacture de tabac dans la ville de Trois-Rivières. Le tableau III ne contient que les produits de la manufacture de Québec. 
les expédient ailleurs dans le Bas-Canada ou dans le Haut-Canada. Ces marchands servent d'intermédiaires entre le gros fournisseur (qui peut se trouver dans la métropole ou aux États-Unis) et le petit commerçant local: ils se trouvent, de plus, en relation directe avec le consommateur par le biais de leur(s) propre(s) magasin(s) de détail.

Un autre type de vente existe: les ventes sur les quais appartenant aux gros marchands-négociants. L'activité commerciale y est intense et encore une fois, la presque totalité des marchandises sont vendues en gros. Ce commerce revêt parfois un aspect assez spécialisé, à cause des marchandises spécifiques qu'on peut y vendre. La Gazette de Québec, tout au cours de la période 1820-1830, nous renseigne là-dessus. Par exemple, une annonce précise qu'à tel jour et à telle heure au quai Goudie (pour ne nommer que celuilà, qui semble être très important vu le nombre élevé de ventes qui s'y déroulent chaque mois) seront vendues «131 tonnes de Rum d'Antigua et de Ste-Lucie, 71 barriques de mélasse, 25 caisses de chocolat» ${ }^{16}$, ou encore «le corps d'un navire de 380 tonneaux, tous ses agrès, 25 voiles, 2 câbles» ${ }^{17}$.

Que la vente se fasse sur les quais Goudie, Atkinson, Pozer, Quirouet, Tremaine, ou sur d'autres quais, cela ne signifie pas pour autant que les marchandises vendues appartiennent au propriétaire du quai. Dans certains cas, les marchandises sont la propriété d'un autre commerçant qui veut vendre la cargaison immédiatement après son débarquement; dans d'autres circonstances, le stock appartient à un commerçant de la métropole ou d'ailleurs (par exemple de Halifax) qui charge un encanteur de s'occuper de la mise en vente ${ }^{18}$.

Il existe, de plus, dans la ville des commerces un peu plus spécialisés, destinés à la vente d'un produit ou d'une catégorie de produits particuliers. C'est le cas, par exemple, des brasseries, où en plus de produire, le propriétaire peut s'occuper de la mise en marché directe, par le biais de son propre magasin de détail, et de la vente d'une partie de sa production à d'autres commerçants. Il s'agit d'une pratique très courante à l'époque dans les petites fabriques de toutes sortes, surtout de la part des grands marchands-négociants. On peut retrouver rue Saint-Jean des établissements de confiserie, de pâtisserie, des boutiques de fer et de forgeron. John Goudie possède une boulangerie, une boucherie,

\footnotetext{
16 La Gazette de Québec, 9 août 1821.

17 Ibid., 6 janvier 1825.

18 Chinic \& Quirouet, Martin Chinic, Cary \& Co, Melvin \& Bélanger, la firme d'Atkinson, celle de Wurtele \& Fraser semblent être parmi les plus actifs encanteurs à Québec au cours de la période 1820-1830.
} 
en plus, bien sûr, de s'occuper de ses multiples autres activités. John Reinhart écoule une partie de la production de sa manufacture de tabac par l'entremise de sa tabagie et vend le reste aux petits commerçants de la ville et des environs.

N'oublions pas non plus certains commerces qui s'occupent uniquement de produits d'importation. C'est le cas des boutiques spécialisées dans la vente d'instruments de musique, dont la plupart sont importés d'Angleterre. Hund et Seabold, qui tiennent boutique au 38 rue Saint-Jean, vendent, entre autres, des pianos qui, malgré leur prix élevé, semblent avoir la faveur de la bourgeoisie commerçante.

Toujours rue Saint-Jean, en entrant dans le magasin de JeanBaptiste Martinucio, on peut se procurer des estampes et des miroirs importés de France, des thermomètres et des lunettes venant d'Angleterre. Au 26 rue Stanislas, J. Robb offre «un bel assortiment de joailleries, montres d'or et d'argent de grand luxe, et autres bijoux, faits à Londres» ${ }^{19}$. Il n'est pas rare de constater dans l'inventaire après décès d'un marchand-négociant ou d'un professionnel que l'individu possède au moins deux montres en or, mises à part celles de son épouse.

Enfin, pour en terminer avec l'énumération des différentes sortes de commerce, signalons les encans du soir. Malgré la diversité des produits vendus, ce genre de commerce paraît destiné à une catégorie sociale spécifique; ce sont des marchandises de luxe ${ }^{20}$ qui sont mises en vente, et il est plausible d'admettre qu'il prend l'aspect autant d'une rencontre mondaine que d'un vrai négoce. Ce genre d'encans du soir se tient au moins une fois la semaine (la Gazette de Québec en fait l'annonce dans presque chacune de ses parutions).

L'analyse des inventaires des stocks de marchandises des marchands-négociants ainsi que le dépouillement de la Gazette de Québec nous permettent de dégager certaines conclusions. Tout d'abord, on constate que le commerce de gros et de détail se trouve en pleine expansion dans la ville de Québec. Devant un marché en déclin, il est peu probable que les marchands-négociants se seraient permis d'investir dans l'achat massif de marchandises. Cette croissance du commerce est aussi l'indice d'une augmentation relative du pouvoir d'achat d'une certaine couche de la population qui possède les moyens de s'offrir ces produits.

19 La Gazette de Québec, 24 juillet 1820.

20 Un exemple parmi d'autres: la Gazette de Québec du 11 octobre 1821 annonce un encan du soir qui se tiendra à l'Hôtel de l'Union. Les objets en vente: une collection de livres précieux, français et anglais, une caisse d'excellents pistolets, de la vaisselle argentée, des joailleries, etc. 
En second lieu, quelques produits vendus en magasin mettent en relief la présence d'une haute bourgeoisie. En effet, la mise en vente de nombreux produits alimentaires exotiques et d'autres objets de luxe révèle l'existence d'individus fortunés. Nous pouvons citer en exemple, dans le secteur alimentaire, la vente de noix espagnoles à raison de cinq pence la livre, du thé vert de Chine de qualité supérieure, évalué à cinq chelins la livre, des noisettes à six pence la livre, du jambon à huit chelins la livre, et du brandy à cinq chelins le gallon. Dans le secteur de l'habillement, on trouve des tissus comme le satin, la soie, le velours, des mouchoirs et des robes de soie, des écharpes de dentelle, des redingotes de velours de soie, des chaussures et des bottes importées d'Europe. Dans le secteur mobilier, des meubles en bois rare ou exotique provenant d'aussi loin que de l'Espagne, du Brésil ou du Japon; des instruments de musique, tels le violon, le piano, la flûte, etc. Ce sont dans l'ensemble des objets qu'on ne retrace que rarement chez les travailleurs ordinaires ou chez les gens de la campagne ${ }^{21}$.

L'existence du commerce de biens de luxe confirme l'enrichissement des marchands-négociants, suite à l'expansion du commerce du bois, à la chute des prix agricoles, à l'exploitation forestière et à la construction navale. Ainsi, en prenant pour paramètres les dix catégories établies par Jean-Pierre Wallot et Gilles Paquet ${ }^{22}$ dans leur étude sur la structure de la richesse de la population dans la région de Montréal au tournant du XIXe siècle, il ne fait pas de doute que l'enrichissement a surtout profité à une catégorie particulière de la population de la ville.

Enfin, une analyse des inventaires en magasin permet de déceler un marché très actif dans les secteurs de la construction ${ }^{23}$ et de la menuiserie. Un bon indice en est la quantité énorme de clous de toutes dimensions, de la peinture, du goudron, des outils de travail et autres articles reliés à ces deux secteurs que les marchands-

21 À ce sujet, l'équipe dirigée par Gilles Paquet et Jean-Pierre Wallot, à laquelle j'ai participé, a dépouillé, pour la période allant de 1795 à 1835 , plus de 400 inventaires après décès pour la région de Québec. En général, on ne trouve pas chez les habitants et les hommes de métier les produits de luxe dont nous faisons mention ci-haut: aucun piano, aucune peinture, quelques rares livres, souvent des livres de prières ou une bible.

22 Gilles Paquet et Jean-Pierre Wallot, «Les inventaires après décès à Montréal au tournant du XIXe siècle: préliminaires à une analyse», $R H A F, 30,2$ (sept. 1976). Dans cet article, les auteurs ont regroupé les biens mobiliers et autres de la population en une dizaine de catégories, celles-ci étant suivies d'une section portant sur l'argent possédé par l'individu, une autre sur l'argenterie, puis une encore sur l'or, une section pour les créances et enfin une autre pour les biens immobiliers et fonciers. À partir de ces informations très précises, ils ont pu établir le niveau d'enrichissement des groupes sociaux étudiés.

${ }_{23}$ Cependant, nous ne sommes pas en mesure de préciser s'il s'agit de construction navale ou domiciliaire. Probablement s'agit-il des deux, car à cette époque, de 1820 à 1830 , dans la ville de Québec, ces deux secteurs sont très actifs et créateurs d'emplois. Pour la construction navale, voir A. Faucher, Histoire économique et unité canadienne (Montréal, Fides, 1970), 227-254. 
négociants détiennent dans leurs entrepôts ou en magasin ${ }^{24}$. Le secteur de la construction se révèle fort lucratif pour les gros commerçants; la plupart possèdent eux-mêmes ces articles de construction dans leurs magasins ou sont propriétaires de vastes étendues de terres à bois et d'un moulin à scier. C'est sur ces terres qu'ils se procurent en partie le bois dont ils ont besoin à des fins commerciales. D'autres marchands-négociants, en plus de posséder des terres, passent des contrats avec des cultivateurs pour que ceux-ci leur livrent de grosses quantités de bois ${ }^{25}$.

Brièvement, on peut dire à la lumière des renseignements recueillis dans les inventaires et dans la Gazette de Québec que le commerce est florissant à Québec au cours de la période 18201830. Seul le numéraire peut manquer, à certains moments, ce qui peut expliquer partiellement le nombre grandissant d'individus qui, à cette époque précise, achètent à crédit ${ }^{26}$.

\section{II - Le crédit}

Lorsqu'on étudie le commerce de gros et de détail, on ne peut évidemment pas ignorer son corollaire, le système de crédit, qui est en quelque sorte le moteur du commerce pendant la première moitié du XIXe siècle, dans la ville de Québec. Le rôle primordial que joue le crédit à l'intérieur du système commercial n'est pas nouveau et il ne se trouve nullement circonscrit au Bas-Canada ${ }^{27}$.

24 Le stock de marchandises de Michel Borne contient 62200 clous à bardeaux et à planches, et un quintal de clous de cinq, 17 marteaux et autres outils de travail, 734 madriers, 136 planches, 7000 bardeaux et une quantité indéterminée de peinture et de goudron.

25 Nous n'avons pas cru bon de nous attarder sur le commerce du bois, car madame Louise Dechêne l'a déjà étudié pour la région de Québec. Malgré le fait que son étude ne concerne qu'un individu actif dans ce domaine, il n'en demeure pas moins que l'analyse détaillée de la structure du commerce du bois que l'auteur fait à travers l'étude sur William Price, nous permet de nous faire une idée assez générale de ce commerce pour la première moitié du XIXe siècle. Louise Dechêne, «Les entreprises de William Price, 1810-1850», Histoire sociale, 1 (avril 1968). Sur un plan plus étendu, Fernand Ouellet parle abondamment du commerce du bois, en particulier dans son Histoire économique et sociale du Québec, 1760-1850 (Montréal, Fides, 1971, tomes 1 et 2), ainsi que dans Le Bas-Canada, 1791 1840. Changements structuraux et crise (Éditions de l'Université d'Ottawa, 2e éd., Ottawa, 1980), de même que Gilles Paquet et Jean-Pierre Wallot, «Le Bas-Canada au tournant du 19e siècle: une hypothèse», $R H A F, 25,1$ (juin 1971): 39-61.

26 Le lecteur intéressé à suivre l'évolution du commerce pour une période ultérieure consultera avec profit l'étude de Gaétan Gervais, «Le commerce de détail au Canada (18701880)», RHAF, 33, 4 (mars 1980): 521-556.

27 Ainsi, Fernand Braudel a constaté le même phénomène en Europe: «Et le crédit est de plus en plus l'outil indispensable du grand marchand, [son] capital propre, son «principal» [...]". Ce passage, même s'il concerne le XVIIIe siècle, a beaucoup d'analogies avec la situation à Québec entre 1820 et 1830 . Fernand Braudel, Civilisation matérielle, économie et capitalisme, XVe-XVIIIe siècle, Tome 2, Les jeux de l'échange (Armand Colin, Paris, 1979), 338-339. 


\section{A - Le fonctionnement du crédit}

Le système de crédit repose d'abord sur les comptes dus au marchand-négociant par d'autres commerçants pour fourniture de marchandises, ensuite sur le prêt de sommes d'argent, plus ou moins élevées, à des particuliers ou à de petits marchands. Il comprend aussi les reconnaissances de dettes (billets remis par un (des) acheteur(s) à la suite de l'achat de biens de consommation). Généralement, ces prêts ou ces dettes sont identifiés par une obligation; la reproduction synthèse de l'acte ci-dessous nous en fournit une idée.

Jean-Baptiste Blouin, marchand demeurant en la Paroisse de Berthier en ce District de Québec, lequel reconnaît devoir [...] à Étienne Claude Lagueux, écuyer, marchand demeurant en cette ville de Québec [...] la somme de soixante livres du cours actuel de cette Province, pour valeur reçue, marchandises et effets vendus et livrés [...] Le dit Débiteur promet et s'oblige sous l'hypothèque de tous ses biens, payer [...] au dit Étienne Claude Lagueux [...] à la première demande et réquisition, avec l'intérêt au taux actuel sur la dite somme de Soixante livres à compter de ce jour jusqu'à parfait payement $[\ldots]^{28}$

Ainsi donc, l'obligation est un acte passé devant un notaire, par lequel quelqu'un reconnaît s'être endetté envers son créancier.

Le crédit pour la fourniture de marchandises ${ }^{29}$ s'avère très important à plusieurs points de vue. Tout d'abord, il permet aux petits détaillants qui ne disposent pas d'assez d'argent de pouvoir malgré tout approvisionner continuellement leur commerce. De la sorte, les habitants des régions éloignées peuvent trouver en magasin les produits dont ils ont besoin. Pour le marchand-négociant à Québec, cette forme de crédit le met en contact avec la plupart des personnes actives dans le circuit commercial, du petit détaillant au cultivateur. Le crédit constitue également un secteur d'investissement rentable à coefficient de risque relativement bas. Dans tous les cas, le débiteur hypothèque ses biens meubles et immeubles pour garantir le remboursement de sa dette.

Un acte de quittance peut aussi permettre de connaître s'il y a eu crédit. Il s'agit sensiblement du même genre de contrat qu'une obligation, sauf que, dans ce cas, il revient au créancier de reconnaître avoir reçu du débiteur la somme que ce dernier lui devait.

\footnotetext{
28 ANQ-Q, not. L. T. MacPherson, 21-11-1823, Obligation.

29 Cela consiste en la livraison de marchandises par le créancier à son débiteur. C'est le cas par exemple de John Goudie qui doit la somme de $£ 6368 / 3 / 9$ sterling à la Rogerson, Hunter and Co. d'Angleterre, pour marchandises reçues de cette compagnie. Goudie s'engage à rembourser le montant dans un délai de 13 mois avec l'intérêt annuel de $6 \%$. Ce genre de transactions illustre assez clairement ce qu'est le crédit pour fourniture de marchandises. ANQ-Q, not. Archibald Campbell, no 3125, 24-1-1823.
} 
Un troisième document qui identifie le crédit, c'est la section des dettes actives et passives du marchand, qui se trouve dans son inventaire après décès. Cette section, qui n'est en fait que le relevé du livre comptable du marchand, possède une très grande valeur, car elle permet de dresser un rapide bilan provisoire de la situation financière de l'individu. Provisoire, car d'autres actes, comme les obligations et les quittances, peuvent modifier son bilan financier. Ainsi donc, lorsqu'on tente de dresser l'état du crédit complet d'un marchand, la conjonction de plusieurs types d'actes notariés devient nécessaire ${ }^{30}$.

Dans la ville de Québec pour la période allant de 1820 à 1830 , le marchand-négociant constitue l'intermédiaire entre le gros fournisseur, qui demeure bien souvent hors de la province, et le petit commerçant. Ses magasins le mettent aussi en contact avec les petits acheteurs. À tous ces niveaux, quelqu'un doit de l'argent à un autre. Le marchand-négociant paie rarement comptant son fournisseur, qu'il rembourse après l'écoulement total ou partiel du stock sur le marché. Bien entendu, le marchand-négociant paie des intérêts à son fournisseur pour ce retard. Quant au petit commerçant du quartier, du village ou de la campagne, il s'acquittera de sa dette de la même façon que le marchand-négociant le fait envers son fournisseur, c'est-à-dire qu'il paiera plus tard, avec les intérêts sur la somme due. Et enfin, le citoyen ordinaire, le consommateur, lui aussi achète bien souvent à crédit les articles dont il a besoin.

Le système transparaît clairement à la lecture de la section des dettes actives et passives (à l'intérieur des inventaires) du marchand-négociant: le nombre de personnes qui lui doivent de l'argent pour l'achat de produits dépasse bien souvent la centaine. Ainsi, plus de 130 personnes demeurant un peu partout dans la province du Bas-Canada doivent près de $£ 3500$ courant à John Reinhart pour marchandises qu'il leur a fournies ${ }^{31}$. La même constatation vaut pour Michel Borne dont la section des dettes actives est classée par régions ${ }^{32}$, ainsi que pour de nombreux

30 Sur la valeur et la nécessité de l'utilisation de l'inventaire après décès comme instrument servant à l'analyse historique, le lecteur intéressé consultera avec profit: Gilles Paquet et Jean-Pierre Wallot, «Les inventaires après décès à Montréal au tournant du XIXe siècle, préliminaires à une analyse», op. cit., et l'article de Maurice Garden, «Les inventaires après décès: Source globale de l'histoire sociale lyonnaise ou juxtaposition de monographies familiales», Cahiers d'histoire, tome 12 (1967). En ce qui concerne l'utilisation de l'inventaire après décès comme instrument d'analyse économique, on consultera avec intérêt l'article de Louis Michel, «Le livre de compte (1784-1792) de Gaspard Massue, marchand à Varennes», Histoire sociale, 13, 26 (nov. 1980): 369-398.

31 ANQ-Q, not. Louis Panet, no 263, 11-6-1821.

32 ANQ-Q, not. Charles Maxime De Foy, no 1201, 25-1-1830. Dans le cas de Michel Borne, environ 200 personnes lui doivent de l'argent, totalisant $£ 2811$ courant, pour comptes dus pour achat de marchandises. Sa clientèle se retrouve un peu partout, à Québec et sa région, à Montréal, aux Îles-de-la-Madeleine, à la Baie des Chaleurs, à Rivière-du-Loup et au Nouveau-Brunswick. 
autres marchands-négociants. Du sommet à la base de la pyramide, tout le monde est endetté, l'un envers l'autre. Le crédit joue donc un rôle social non négligeable pour l'époque et touche presque toutes les couches de la population. Il faut néanmoins que l'acheteur (en l'occurrence le gros et le petit commerçant) possède certaines garanties, foncières de préférence.

En ce qui concerne le prêt d'argent, les clauses sont à peu près les mêmes que pour les obligations sur des marchandises. Le résumé qui suit le démontre très bien:

John Goudie residing in the City of Quebec, Merchant \& Ship Builder, $[\ldots]$ by these presents doth declare, acknowledge and confess to owe and to be justly and truly indebted into Peter Burnett [marchand], of the said City of Quebec [...] and accepting hereof in the sum of Five Hundred Pounds current Money of the said Province of Lower Canada [... ${ }^{33}$

Le débiteur hypothèque toujours ses biens meubles et immeubles pour garantir la somme empruntée. L'intérêt de $6 \%$ sur le capital est payable chaque année à date fixe. Le remboursement s'effectue toujours en plusieurs versements: le plus court délai pour remettre l'argent est de six mois, et le plus long, de cinq ans. Mais, généralement, la moyenne pour les remboursements se situe entre un an et demi et trois ans. Le marchand-négociant, contrairement aux banques et aux autres sociétés, n'exige pas nécessairement un endosseur: il se contente de garanties hypothécaires et, généralement, d'un bien foncier.

Le crédit constitue une source de revenus non négligeable pour le marchand-négociant de la ville de Québec, au cours de la période 1820-1830. L'endettement de la population envers ce dernier provient principalement de l'achat de biens de consommation courante ou de l'acquisition de biens durables. Le marchandnégociant agit, en quelque sorte, comme le banquier des gens ordinaires, en ce qui concerne le prêt d'espèces. En effet, une analyse des livres de comptes de la Banque de Québec, pour la période allant de 1820 à 1831, montre qu'aucun citoyen ordinaire (par exemple, un journalier, un charpentier, un artisan ou un cultivateur, etc.) ne détient de compte dans cette institution ${ }^{34}$. Cette banque ne prête qu'aux personnes fortunées ou à celles ayant un revenu élevé.

\footnotetext{
33 De plus, John Goudie convient et promet de rembourser son emprunt d'argent à la première demande de son créancier. L'intérêt sur le montant est de $6 \%$ par année. ANQ-Q, not. Archibald Campbell, no 3157, 31-3-1823, Obligation. $/ 5, / 6, / 7)$.

ANQ-Q, Banque de Québec, Ledger, Oct. 1820 à janv. 1831 (P0184-/2,/3,/4,
} 
Il faut également noter qu'il y a une tendance générale à ajouter un intérêt de $6 \%$ sur la somme due ${ }^{35}$. L'analyse des actes ne nous a pas permis de déceler de pratiques usurières. Par contre, il se peut qu'il y ait eu des ententes verbales entre le prêteur et le débiteur pour un taux d'intérêt plus élevé. Cependant, certaines formes de remboursement peuvent remplacer ou suppléer le taux d'intérêt de 6\%, par exemple, le remboursement d'une somme en marchandises ou en une terre et en produits agricoles.

\section{B - Les créanciers des marchands}

Nous avons précédemment mentionné que le système commercial repose sur le crédit; ainsi, du fournisseur au détaillant, en passant par le consommateur, tous sont endettés auprès de leurs prêteurs. Le marchand-négociant n'échappe pas non plus à l'endettement.

Les créanciers du marchand-négociant lui avancent des fonds pour l'achat de grosses quantités de marchandises ou lui prêtent parfois directement de l'argent pour l'acquisition d'un bien important, comme un navire, une terre ou un immeuble. Dans toutes ces situations, les sommes d'argent engagées sont relativement élevées, comme on peut le constater à l'aide du tableau IV.

\section{TABLEAU IV}

\section{Répartition des créances des marchands-négociants en fonction des sommes dues \\ 1820-1830 ${ }^{1}$}

$£ 300-1099 £ 1100-3099 £ 3100-5099 £ 5100$ et plus

\begin{tabular}{lrrrr}
\hline Banque $^{2}$ & $17 \%$ & $30 \%$ & $47 \%$ & $21 \%$ \\
Société $^{3}$ & $7 \%$ & $25 \%$ & $11 \%$ & $20 \%$ \\
Particulier $^{4}$ & $76 \%$ & $45 \%$ & $42 \%$ & $59 \%$ \\
\hline Total & $100 \%$ & $100 \%$ & $100 \%$ & $100 \%$ \\
\hline
\end{tabular}

Légende: ${ }^{1}$ Le tableau repose sur l'analyse d'une centaine d'actes notariés comprenant des obligations et les dettes passives des 20 marchands-négociants de l'échantillon.

${ }^{2}$ Banque de Québec; Banque de Montréal, Banque du Canada.

${ }^{3}$ Des sociétés privées, telles la Quebec Fire Assurance, la Rogerson, Hunter and Co., Chinic et Quirouet.

${ }^{4}$ Essentiellement des marchands-négociants.

35 C'est en 1777 que le taux légal de l'intérêt est fixé à 6\% par année. Statuts provinciaux, 1777, Geo. 111 , c.3. 
Le tableau IV nous apprend que les créanciers ou les bailleurs de fonds du marchand-négociant se révèlent être, dans la majorité des cas, d'autres marchands-négociants. Pour les sommes allant de $£ 300$ à $£ 1099$ courant, le marchand-négociant préfère, dans une proportion de $76 \%$, s'adresser à ses homologues; les banques viennent au second rang, et, très loin derrière, les sociétés (elles-mêmes dirigées par des marchands-négociants). On doit remarquer que la proportion diminue sensiblement pour la fourchette allant de $£ 1100$ à $£ 3099$ courant, car, à ce stade, les banques talonnent de près les particuliers. Pour les sommes entre $£ 3100$ et $£ 5099$ courant, les banques constituent la première source de financement.

De toute façon, les créanciers qui financent les marchandsnégociants sont, directement ou indirectement, dans une proportion de presque $98 \%$, d'autres marchands-négociants. Prenons le cas des banques. Les conseils d'administration de la Banque de Québec et de la succursale de Québec de la Banque de Montréal, au cours de la période $1820-1830$, sont composés presque exclusivement de marchands-négociants très actifs sur le plan des affaires. Ceci vaut également pour les sociétés qui, bien souvent, ne sont que la réunion de deux ou trois marchands-négociants qui font des affaires ensemble. De plus, si le tableau IV indique un pourcentage très faible de participation des sociétés comme créanciers des marchands-négociants, soit respectivement $7 \%, 25 \%$, $11 \%$ et $20 \%$, ceci ne signifie pas qu'elles s'avèrent inactives dans ce domaine. Nous croyons, après avoir consulté de nombreux actes, que ces sociétés ont plutôt tendance à brasser des affaires avec des petits commerçants.

Les relations étroites entre marchands-négociants peuvent en partie expliquer les raisons qui poussent ces hommes d'affaires à s'adresser à leurs semblables pour obtenir de l'argent ou des marchandises. Ces relations peuvent prendre de multiples formes: il peut s'agir de liens de parenté, d'affaires, de liens sociaux et ethniques. Les marchands-négociants finissent par bien se connaître et par constituer un réseau d'affaires. D'ailleurs, ils sont presque les seuls à posséder les ressources financières. En outre, si les marchands dans l'ensemble sont plus réticents à emprunter des institutions bancaires, il faut ajouter aux raisons déjà citées le manque de tradition bancaire au sein de la communauté des affaires. Les banques sont encore bien jeunes: la création de la Banque de Québec ne remonte qu'à 1818 et son incorporation, à 1822 . Quant à la Banque de Montréal, fondée en 1817, sa première succursale à Québec date aussi de 1818. Finalement, il convient d'ajouter un autre élément qui pourrait inciter les marchands à emprunter ailleurs qu'à la banque: l'endossement. En effet, les banques exigent 
souvent un endosseur pour le client désireux d'obtenir un prêt, et comme les sommes en jeu sont généralement élevées, on peut croire qu'il n'est pas facile à l'emprunteur de trouver une ou des personnes prêtes à garantir les emprunts, car il y a toujours le danger que le débiteur ne puisse pas honorer ses engagements.

Le système de crédit, selon les considérations mentionnées ci-haut, est un phénomène personnalisé beaucoup plus qu'institutionnalisé; dans plus de $60 \%$ des cas, ce sont des individus qui s'endettent envers d'autres individus, plutôt qu'envers les banques. Cela demeurera la caractéristique principale du crédit dans la ville de Québec durant toute la première moitié du XIXe siècle.

\section{C-Le profil du débiteur}

Avant d'étudier les divers modes de remboursement, nous nous devons de souligner qu'il est difficile d'établir un profil net du marchand débiteur. Lorsqu'on analyse les données du tableau $\mathrm{V}$, à la section «dette passive», on s'aperçoit que la plupart des marchands représentés dans notre échantillon sont endettés. Ainsi, la moyenne est de $£ 13411$ courant par marchand, ce qui représente un montant assez élevé comparativement à la moyenne de $£ 4896$ courant pour la dette active. Ce constat d'endettement, malgré son caractère temporaire, tend à confirmer que les grands marchands se servent abondamment du crédit, lequel joue un rôle vital dans l'économie capitaliste de type commercial dans la ville de Québec entre 1820 et 1830.

Une autre constatation que nous tirons du tableau $\mathrm{V}$, c'est le nombre réduit de créanciers par débiteur. En effet, plus la somme due est élevée, plus le nombre de créanciers diminue. Ainsi, nous avons pu établir que pour les montants supérieurs à $£ 1000$ courant, la moyenne est de cinq créanciers par débiteur ${ }^{36}$. Le tableau $\mathrm{V}$ permet de voir aussi qu'on est redevable envers les marchands. Comme on peut le constater, $100 \%$ de ceux qui sont retenus dans notre échantillon présentent une dette active. Cependant, le point important à retenir demeure la relative primauté de la dette passive sur la dette active, ce qui entraîne dans $67 \%$ des cas un solde négatif, comparativement à $33 \%$ qui présentent un solde positif.

Si crédit est synonyme d'endettement, ce dernier n'implique pas nécessairement la faillite. En effet, bien que la plupart des

36 Par exemple, $61 \%$ de l'ensemble de la dette passive de Thomas White est dû à seulement trois personnes; dans le cas de James McCallum, 54\% du total de ses dettes va à quatre autres marchands. $59 \%$ du total de la dette passive de John Goudie va à sept créanciers. Fait significatif: à l'intérieur de ces pourcentages, le nombre de créanciers se réduit encore. Ainsi, pour McCallum, 28\% du 54\% est dû à George Pozer; dans le $59 \%$ que doit Goudie, $14 \%$ va à Pozer. 


\section{TABLEAU V}

État du crédit des marchands dette active et passive en $£$ courant, Québec

\section{0-1830}

\begin{tabular}{|c|c|c|c|}
\hline Marchands & Dette active & Dette passive & Solde $(+$ ou -$)$ \\
\hline Thomas Langlois & $1083 / 2 / 9$ & $61 / 10 / 0$ & $+1021 / 12 / 9$ \\
\hline Joseph Jones & $9631 / 7 / 2$ & $532 / 14 / 0$ & $+9098 / 13 / 2$ \\
\hline Thomas White & $3339 / 2 / 0$ & $8256 / 6 / 0$ & $-4917 / 4 / 0$ \\
\hline Robert Wood & $5883 / 9 / 6$ & $23462 / 5 / 6$ & $-17578 / 16 / 0$ \\
\hline James McCallum & $5755 / 6 / 0$ & $58674 / 5 / 5$ & $-52918 / 19 / 5$ \\
\hline John Alexander & $818 / 2 / 0$ & $845 / 13 / 7$ & $-\quad 27 / 11 / 7$ \\
\hline Michel Borne & 2811 & 1658 & +1153 \\
\hline Frederick Limpp & $3378 / 2 / 7$ & $5405 / 13 / 7$ & $-2027 / 11 / 0$ \\
\hline John Goudie & 4900 & 24340 & -19440 \\
\hline Jacob Pozer & 633 & 36431 & -35798 \\
\hline John Reinhart & 6326 & 9502 & -3176 \\
\hline John McCord & 625 & 916 & $-\quad 291$ \\
\hline Pierre Pelletier & 1743 & 46 & +1697 \\
\hline Martin Chinic & 26020 & 24947 & +1073 \\
\hline Charles Stuart & 502 & $6085 / 16 / 7$ & $-5583 / 16 / 7$ \\
\hline Total: 15 & \multicolumn{3}{|c|}{$\begin{array}{l}£ 73 \quad 448 / 12 / 0 \quad £ 201 \quad 164 / 4 / 8 \\
(1762766 \# 8 \text { sols })(4827941 \# 12 \text { sols })\end{array}$} \\
\hline Moyenne & $\begin{array}{l}£ 4896 \\
(117504 \#)\end{array}$ & $\begin{array}{l}£ 13411 \\
(321864 \#)\end{array}$ & \\
\hline
\end{tabular}

Légende: $\mathrm{Le}+$ indique que le solde est positif; le - , que le solde est négatif. Les données pour les 5 autres marchands qui n'apparaissent pas dans le tableau sont incomplètes.

Source: Les minutiers des notaires aux ANQ-Q.

marchands-négociants se trouvent souvent très endettés, il ne faut surtout pas croire que dans ce milieu on «tire le diable par la queue». Ces commerçants ont généralement en leur possession beaucoup d'argent. De plus, ils possèdent des actions négociables qui proviennent des banques, des compagnies d'assurances et des compagnies maritimes. Â cela s'ajoutent des biens immobiliers et fonciers qui suffiraient à effacer toute dette urgente.

\section{$D$ - Les modes de remboursement du crédit}

À l'analyse des modalités du remboursement, on s'aperçoit que les créanciers des marchands-négociants leur accordent suffi- 
samment de temps pour effacer leurs dettes. Premièrement, s'il s'agit d'un prêt direct d'argent (concrétisé par une obligation passée devant notaire ou par un billet de reconnaissance de dette, qui lui ne nécessite pas la présence du notaire), la période de remboursement s'étale sur un plus long terme que celui offert pour le remboursement de marchandises reçues. Le remboursement se fait entre trois et cinq ans, parfois plus, mais va rarement au-delà de cinq années, à moins que le prêt ne se transforme en rente.

Deuxièmement, dans le cas d'une obligation pour la fourniture de marchandises, il est très rare que le délai de paiement dépasse trois ans; il se situe généralement entre six mois et deux ans. En général, le délai de remboursement pour l'argent ne commence qu'après une année, et, pour les fournitures de stock, le remboursement n'excède pas 14 mois. En ce qui concerne le crédit pour de l'argent, le créancier peut exiger son remboursement à la première demande. On rencontre fréquemment cette condition, c'est-à-dire que le débiteur s'engage à rendre l'argent dès que son créancier en fera la demande, sans pour autant arrêter de verser l'intérêt annuel de $6 \%$ d'ici là. Si l'on considère que, bien souvent, les sommes concernées sont assez élevées (de $£ 300$ à $£ 5000$ courant), il est probable que le créancier ne se fait pas rembourser dans un très court délai. Ce genre de prêt peut aussi se transformer en rente. Le débiteur versera à chaque année l'intérêt sur le capital, sans pour autant le rembourser.

\section{E - Ethnicité et crédit}

En ce qui concerne l'origine ethnique des créanciers, les données parlent d'elles-mêmes; les marchands-négociants canadiensanglais sont plus présents que leurs homologues canadiens-français qui possèdent moins de capitaux. À la Banque de Québec, ces derniers ne dépasseront le chiffre de $40 \%$ de l'ensemble des administrateurs qu'en 1823, et leur présence diminue tout au cours de la période, comme le met en évidence le tableau VI. Ils ne comptent plus que pour $14 \%$ du total des administrateurs en 1830. Par ailleurs, à la succursale de Québec de la Banque de Montréal, la présence canadienne-française au sein du comité de gérance est presque symbolique. En effet, en 1821 et 1822 , un seul administrateur sur onze est canadien-français, et durant le reste de la période, aucun n'y est représenté.

Cette relative faiblesse du milieu d'affaires canadien-français à l'intérieur des institutions financières peut partiellement s'expliquer (nous disons partiellement parce que les raisons politiques sont très connues et que l'historiographie en fait mention abondamment) en relevant la proportion des détenteurs d'actions dans 


\section{TABLEAU VI}

\section{Répartition ethnique (en \%) des administrateurs des banques à Québec entre 1820 et 1830}

\begin{tabular}{|c|c|c|c|c|}
\hline \multirow[t]{2}{*}{ Années } & \multicolumn{2}{|c|}{ Banque de Québec } & \multicolumn{2}{|c|}{ Banque de Montréal } \\
\hline & $\mathrm{CF}$ & $\mathrm{CA}$ & CF & C A \\
\hline 1820 & 29 & 71 & 0 & 100 \\
\hline 1821 & 38 & 62 & 9 & 91 \\
\hline 1822 & 38 & 62 & 9 & 91 \\
\hline 1823 & 43 & 57 & 0 & 100 \\
\hline 1824 & 38 & 62 & 0 & 100 \\
\hline 1825 & 36 & 64 & 0 & 100 \\
\hline 1826 & 36 & 64 & 0 & 100 \\
\hline 1827 & 29 & 71 & 0 & 100 \\
\hline 1828 & 21 & 79 & 0 & 100 \\
\hline 1829 & 14 & 86 & 0 & 100 \\
\hline 1830 & 14 & 86 & 0 & 100 \\
\hline
\end{tabular}

Légende: $\mathrm{C} \mathrm{F}$ : Canadiens français

C A : Canadiens anglais

Source: Les Almanachs de Québec, de 1820 à 1830.

1 Les administrateurs de la Banque de Québec sont, pour la plupart, des détenteurs d'actions de cette institution. Et, on peut croire qu'ils décident (après avoir obtenu probablement le consentement des autres souscripteurs) des grandes orientations de la banque, ainsi que de son administration quotidienne. Le conseil d'administration est composé d'un président, d'un vice-président et d'une dizaine de directeurs. Par ailleurs, à la Banque de Montréal (succursale de Québec), les administrateurs sont des gérants qui reçoivent leurs directives de Montréal. En effet, les principaux détenteurs d'actions, ainsi que le siège social de cette banque se trouvent à Montréal. À Québec, l'administration est assumée par un président et des directeurs.

ces différentes institutions. Lors de la création de la Banque de Québec en $1818,61 \%$ de ceux qui ont acheté les 3000 parts à $£ 25$ courant chacune pour former le capital sont des Canadiens anglais et $39 \%$ sont des Canadiens français ${ }^{37}$. La composition du conseil d'administration reflétera donc la composition ethnique, ce qui explique que les hommes d'affaires canadiens-anglais contrôlent et administrent la Banque de Québec. Leur emprise s'accroît entre

37 Les Statuts provinciaux du Bas-Canada, vol. 11, Acte pour incorporer certaines personnes y mentionnées, sous le nom de «Banque de Québec», A.D. 1822, c.26. Si en 1818 la proportion de Canadiens français qui détiennent des actions de la Banque de Québec est de $39 \%$, elle chute à $19 \%$ en 1830 , d'après les calculs de F. Ouellet, Le Bas-Canada, 17911840. Changements structuraux et crise, op. cit., 210. 
1820 et 1830 grâce à ce qui nous semble être une stratégie de rachat systématique des actions possédées par le groupe francophone.

La représentation des marchands canadiens-français n'est pas plus forte dans l'administration de la succursale de la Banque de Montréal, à Québec. Encore là, il faut en chercher les causes lors de la création de cette banque. Le pourcentage de Canadiens français possédant des actions s'avère trop faible ${ }^{38}$ pour que ceux-ci puissent exercer une influence quelconque sur la composition du comité de gérance.

Ainsi, au niveau des banques qui deviennent les créancières des marchands-négociants, l'élément anglais dispose d'une nette avance sur l'élément francophone. La situation s'avère à peu près la même pour les créanciers individuels; c'est ce que nous avons pu constater à l'analyse de la section des dettes passives et actives de l'ensemble des marchands de notre échantillon ${ }^{39}$. À notre avis, il est important de retenir l'hypothèse de la monopolisation du gros crédit par l'élément anglophone de la ville de Québec pour la période 1820-1830.

\section{CONCLUSION}

Nous sommes conscient de ne pas avoir épuisé un sujet aussi complexe que le commerce et le crédit à Québec au cours de la période 1820-1830. Notre préoccupation était avant tout de jeter quelques balises dans le but de faire progresser le débat. Ainsi, il serait souhaitable que d'autres chercheurs qui se pencheront sur ces sujets utilisent des sources différentes de celles que nous employons ou qu'ils approfondissent nos données, afin de susciter de nouvelles discussions.

Notre démarche nous a permis d'évoquer la dynamique du commerce de gros et de détail, et du crédit dans la capitale bascanadienne, entre 1820 et 1830 . Période-clé dans la mesure où les mutations socio-économiques se précisent. De plus, cette étude nous présente une société où le marché s'impose et où le crédit per-

38 Les Statuts provinciaux du Bas-Canada, 11 (1822): c. 25.

39 Par exemple, pour les sommes supérieures à $£ 300$ courant, dues par John Goudie, aucune n'est due à un Canadien français. Parmi tous les créanciers de Jacob Pozer, un seul porte un nom francophone, soit Charles Jourdain, et la seule société canadiennefrançaise envers laquelle il soit endetté - pour $£ 450$ courant - se nomme Chinic et Quirouet. La même constatation vaut pour Frederick Limpp dont le manufacturier John Reinhart constitue le principal créancier. John Mure, un autre marchand-négociant, ne compte aucun Canadien français au nombre de ses débiteurs, pour des sommes supérieures à $£ 300$ courant. Ainsi, pour la période 1820-1830, nous n'avons rencontré que quelques Canadiens français à qui l'on doive plus de $£ 1000$ courant, entre autres, Charles Jourdain et François Le Houllier. Chez le marchand Thomas White, toutes les dettes s'établissant entre $£ 1000$ et $£ 2000$ courant vont à des Canadiens anglais. 
met à la population une plus large accessibilité aux biens de consommation disponibles.

En outre, il ressort clairement que les activités économiques de la ville sont contrôlées par un groupe restreint d'individus qui exercent de fait une situation de monopole, en particulier sur le crédit. Enfin, cet aperçu permet également de constater une stratification sociale certaine. En effet, cette mainmise sur l'économie de la ville par le biais de la diversification des activités économiques des marchands-négociants met en place les germes d'une société où le capital et le marché deviennent dominants et où les antagonismes de classes commencent déjà à se manifester. 